\title{
CORRESPONDENCE
}

\section{LGI1 positive paraneoplastic limbic encephalitis: A case report of atypical presentation}

Limbic encephalitis is a rare disorder mainly affecting the medial temporal lobe and is classically paraneoplastic. ${ }^{1}$ Autoimmune etiologies also exist, such as antibodies against leucine-rich glioma activated 1 (LGI1). Most cases of anti-LGI1 encephalitis are not associated with tumors. ${ }^{2}$ Subacute memory loss is the predominant feature, and most patients develop focal seizures, especially faciobrachial dystonic seizures (FBDSs). ${ }^{1,2}$ Immunotherapies usually show a good response, but are less effective in paraneoplastic cases. ${ }^{3}$ We report a case of steroid-responsive anti-LGI1 encephalitis with atypical presentations of sensory aphasia during relapse from rectal carcinoma, an atypical site.

\section{Case Report}

A 70-year-old man presented with tenesmus, small caliber stool, anal bleeding, and body weight loss of $13 \mathrm{~kg}$ within 1 year. A subsequent study revealed an elevated carcinoembryonic antigen (CEA) level of $22.1 \mathrm{ng} / \mathrm{mL}$, and colonoscopy showed an obstructive mass of $10 \mathrm{~cm}$ into the anal verge. A biopsy report revealed a rectal adenocarcinoma, and the initial staging was cT3N1M0. Neoadjuvant chemoradiotherapy was started after the pathological diagnosis was confirmed. Neuropsychiatric symptoms developed soon after starting neoadjuvant chemo-radiotherapy, including memory impairment, visual hallucinations, confusion, and sleep disturbance. Initial brain magnetic resonance imaging (MRI) was performed 3 weeks after the onset of cognitive impairment, which revealed a T2-hyperintense lesion in the left medial temporal lobe, and paraneoplastic limbic encephalitis was suspected (Figure 1, A and D). Both cerebrospinal fluid (CSF) analysis and an electroencephalography (EEG) study were normal, however LGI1 antibodies were detected in both serum (1:10+) and CSF (1:1+). His symptoms improved spontaneously over a period of 6 weeks.

About 1 month later, he suffered from language impairment with alexia and agraphia. He failed to understand all forms of communication, including verbal, written, pictures, and gestures. While he could speak fluently and comprehensively, the content was irrelevant to the conversation. The language deficit resembled complete sensory aphasia and he was unaware of the deficit. Repeat brain MRI showed an extended left medial temporal lobe lesion (Figure 1, B and E). Intravenous pulse steroid therapy was administered with methylprednisolone $1000 \mathrm{mg} /$ day for 5 days, and the language deficit completely resolved. Follow-up brain MRI 1 month later showed regressive changes (Figure 1, C and F). Robotic lower anterior resection of the tumor was then performed, and the pathology revealed a $2.0 \times 1.8 \times 1.0 \mathrm{~cm}$ well-differentiated rectal adenocarcinoma with post-treatment staging of ypT3N1aMx. Immunohistochemistry showed no loss of nuclear expression of mismatch repair protein, indicating a low probability of microsatellite instability.

No further cognitive or neurological deterioration was observed during 1 year of follow-up. No seizures occurred and no prophylactic antiepileptic drugs were given during the entire course, however mild residual memory impairment and sleep disturbance were noted. Cognitive function assessments showed a Mini-Mental State Examination score of 29 and a Clinical Dementia Rating of 0.5, indicating minimal residual cognitive impairment.

Anti-LGI1 encephalitis is a rare disorder that more frequently occurs in elderly males, with a mean onset age of 60 years. ${ }^{2}$ Only about $11 \%$ of anti-LGI1 encephalitis cases are associated with tumors, with thymoma and lung cancer being the most common and colorectal cancer rarely being reported. ${ }^{2,4,5}$ In a case series of 57 patients with limbic encephalitis and antibodies against LGI1, no colon or rectal tumors were found in six patients who had tumors. ${ }^{6}$ A similar finding was found in another study of 68 patients with LGI1 antibodies, in which no colorectal tumors were found in nine patients who had tumors. ${ }^{7}$ Tumors of colon or rectal origin have been reported in isolated cases. Rectal carcinoma in situ was detected in one patient among four tumor-associated cases in a long-term follow-up study of 36 patients $^{3}$, and a study of 76 patients with LGI1 antibody-related cognitive impairment reported one case of colon carcinoma in five patients who had tumors. ${ }^{8}$ In another review of anti-LGI1/CASPR2 seropositive patients, rectal carcinoma was found in one of three patients with tumors. ${ }^{9}$ 

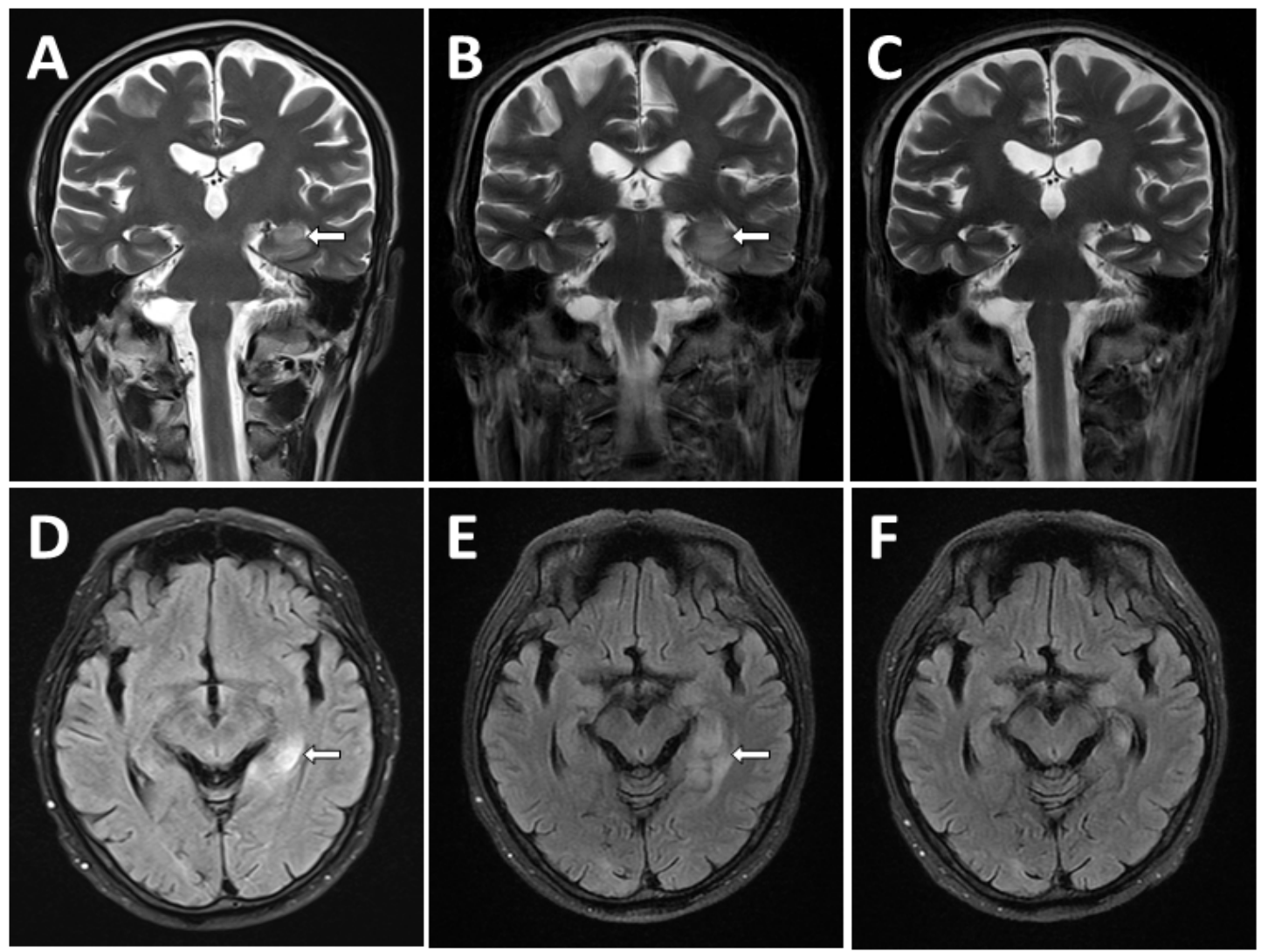

Figure 1. MRI showing a left medial temporal lobe lesion. (arrows)

A, B, C: T2-weighted imaging, coronal view. D, E, F: T2-weighted fluid-attenuated inversion recovery, axial view.

A, D: 3 weeks after symptom onset; B, E: symptom progression after 1 month; C, F: 1 month after pulse steroid therapy

Almost all patients with anti-LGI1 encephalitis experience subacute memory loss and spatial disorientation ${ }^{2,3}$, and FBDSs and subtle focal seizures, including subtle autonomic or dyscognitive seizures, usually occur prior to memory loss. ${ }^{2,3,5}$ More than half of patients develop generalized tonicclonic seizure later in the disease course..$^{2,3}$ Behavioral changes are also common, including apathy, irritability, disinhibition, and compulsive behavior. ${ }^{2,3}$ Rapid eye movement sleep behavior disorder is another common feature. ${ }^{1,2,3}$ In addition, hyponatremia has been reported in $60 \%$ of patients, possibly due to the inappropriate secretion of antidiuretic hormone induced by LGI1 expression in the hypothalamus and kidneys. ${ }^{1}$

CSF analysis is typically normal ${ }^{2}$, however antibodies to LGI1 can be detected in both serum and CSF. Testing both serum and CSF is recommended, although serum testing is generally more sensitive. ${ }^{2,4,5}$ Brain MRI may show T2 hyperintensity in the medial temporal lobe, typical for limbic encephalitis. ${ }^{2,5,10}$ These lesions evolve into medial temporal sclerosis in nearly half of cases in the long-term. ${ }^{3,10} \mathrm{~T} 2$ hyperintensities in the basal ganglia may be found in patients with FBDSs. ${ }^{2,5}$

Immunotherapies, including pulse steroid therapy, intravenous immunoglobulin, and plasma exchange have been reported to be effective in $80 \%$ of patients with anti-LGI1 limbic encephalitis, but less effective in paraneoplastic cases. ${ }^{1,3}$ The reported median time for treatment response is 2 weeks, and the median time for recovery is 33 weeks. ${ }^{3}$ The use of immunosuppressive agents such as cyclophosphamide and rituximab has also been reported. ${ }^{1}$ Searching for a tumor is suggested in patients who do not respond to immunotherapy. For anti-LGI1 encephalitis associated with tumors, both tumor therapy and immunotherapy are needed to achieve the best outcome. ${ }^{1,3}$ The long-term outcome has been reported to be favorable in $67 \%$ of cases, but relapses are common and occur in about $35 \%$ 
of patients. ${ }^{3}$ The most common residual deficits are mild amnesia and spatial disorientation. ${ }^{3}$ Sleep disturbance may also persist.

In conclusion, due to the rare nature and wide range of non-specific neuropsychiatric symptoms, anti-LGI1 encephalitis is considered a diagnostic challenge and the median treatment delay is 25 weeks. ${ }^{3}$ The patient in the current report had rectal cancer and sensory aphasia during relapse of limbic encephalitis. Rectal cancer has rarely been associated with anti-LGI1 encephalitis, and sensory aphasia has never been reported. Immunotherapy has been reported to be less effective in tumor-associated cases, and our patient showed a rapid response to pulse steroid therapy. No relapses occurred after tumor resection. We hypothesize that an early diagnosis, timely treatment, and prompt tumor therapy contribute to a favorable outcome, as in our case. This case highlights the need to identify atypical features of anti-LGI1 encephalitis and reduce the delay to treatment.

\section{${ }^{1}$ Chien-Chung Cheng $M D,{ }^{1,2,3}$ Jia-Ying Sung $M D P h D,{ }^{1,2,3}$ Chih-Shan Huang $M D$}

${ }^{1}$ Department of Neurology, Wan Fang Hospital, Taipei Medical University, Taipei, Taiwan; ${ }^{2}$ Taipei Neuroscience Institute, Taipei Medical University, Taipei, Taiwan; ${ }^{3}$ Department of Neurology, School of Medicine, College of Medicine, Taipei Medical University, Taipei, Taiwan

Keywords: Leucine-rich glioma-inactivated (LGI1), paraneoplastic, limbic encephalitis, rectal adenocarcinoma

Address correspondence to: Chih-Shan Huang, Department of Neurology, Wan Fang Hospital, Taipei Medical University, No.111, Sec. 3, Xinglong Rd., Wenshan Dist., Taipei City 116, Taiwan. Tel: +886-2-29307930 ext 6940, E-mail: alexcheng79@gmail.com, sonichuang@yahoo.com

Date of Submission: 10 July 2019; Date of Acceptance: 14 September 2021

https://doi.org/10.54029/2021auz

\section{REFERENCES}

1. Bhardwaj K, Sharma SK, Pandey AK, et al. A Case of Limbic Encephalitis: Antibody LGI1 Associated Encephalitis. J Neurol Neurosci 2016; 7:4.

2. Bastiaansen AEM, van Sonderen A, Titulaer MJ. Autoimmune encephalitis with anti-leucine-rich glioma-inactivated 1 or anti-contactin-associated protein-like 2 antibodies (formerly called voltage-gated potassium channel-complex antibodies). Curr Opin Neurol 2017; 30:302-9.

3. van Sonderen A, Thijs RD, Coenders EC, et al. Anti-LGI1 encephalitis: clinical syndrome and long-term followup. Neurology 2016; 87:1449-56.

4. Rosenfeld MR, Dalmau J. Anti-NMDA-receptor encephalitis and other synaptic autoimmune disorders. Curr Treat Options Neurol 2011; 13(3):324-32.

5. van Sonderen A, Schreurs MWJ, Wirtz PW, et al. From VGKC to LGI1 and Caspr2 encephalitis: The evolution of a disease entity over time. Autoimmun Rev 2016; 15(10): 970-4.

6. Lai M, Huijbers MG, Lancaster E, et al. Investigation of LGI1 as the antigen in \& limbic encephalitis previously attributed to potassium channels: a case series. Lancet Neurol 2010; 9:776-85.

7. Binks S, Varley JA, Lee W, et al. Distinct HLA associations of LGI1 and CASPR2-antibody diseases. Brain 2018; 141:2263-71.

8. Arino H, Armangue T, Petit-Pedrol M, et al. Anti-LGI1-associated cognitive impairment: presentation and longterm outcome. Neurology 2016; 87:759-65.

9. Yeo T, Chen Z, Yong KP, Wong PYW, Chai JYH, Tan K. Distinction between anti-VGKC-complex seropositive patients with and without anti-LGI1/CASPR2 antibodies. J Neurol Sci 2018; 391:64-71.

10. Kotsenas AL, Watson RE, Pittock SJ, et al. MRI findings in autoimmune voltage-gated potassium channel complex encephalitis with seizures: one potential etiology for mesial temporal sclerosis. Am J Neuroradiol 2014; 35:84-9. 